\title{
Different Chondrogenic Potential among Human Induced Pluripotent Stem Cells from Diverse Origin Primary Cells
}

\author{
Yeri Alice Rim $\mathbb{D}^{1,2}$ Yoojun Nam, ${ }^{1,2}$ Narae Park $\left(D,{ }^{1,2}\right.$ Hyerin Jung, ${ }^{1,2}$ Yeonsue Jang, ${ }^{1,2}$ \\ Jennifer Lee, ${ }^{1,2}$ and Ji Hyeon Ju $\mathbb{1}^{1,2}$ \\ ${ }^{1}$ Catholic iPSC Research Center, College of Medicine, The Catholic University of Korea, Seoul 137-701, Republic of Korea \\ ${ }^{2}$ Division of Rheumatology, Department of Internal Medicine, Seoul St. Mary's Hospital, Institute of Medical Science, \\ College of Medicine, The Catholic University of Korea, Seoul 137-701, Republic of Korea
}

Correspondence should be addressed to Ji Hyeon Ju; juji@catholic.ac.kr

Received 28 July 2017; Revised 9 October 2017; Accepted 16 October 2017; Published 21 January 2018

Academic Editor: Celeste Scotti

Copyright ( 2018 Yeri Alice Rim et al. This is an open access article distributed under the Creative Commons Attribution License, which permits unrestricted use, distribution, and reproduction in any medium, provided the original work is properly cited.

\begin{abstract}
Scientists have tried to reprogram various origins of primary cells into human induced pluripotent stem cells (hiPSCs). Every somatic cell can theoretically become a hiPSC and give rise to targeted cells of the human body. However, there have been debates on the controversy about the differentiation propensity according to the origin of primary cells. We reprogrammed hiPSCs from four different types of primary cells such as dermal fibroblasts (DF, $n=3$ ), peripheral blood mononuclear cells (PBMC, $n=3$ ), cord blood mononuclear cells (CBMC, $n=3$ ), and osteoarthritis fibroblast-like synoviocytes (OAFLS, $n=3$ ). Established hiPSCs were differentiated into chondrogenic pellets. All told, cartilage-specific markers tended to express more by the order of CBMC $>$ DF $>$ PBMC $>$ FLS. Origin of primary cells may influence the reprogramming and differentiation thereafter. In the context of chondrogenic propensity, CBMC-derived hiPSCs can be a fairly good candidate cell source for cartilage regeneration. The differentiation of hiPSCs into chondrocytes may help develop "cartilage in a dish" in the future. Also, the ideal cell source of hiPSC for chondrogenesis may contribute to future application as well.
\end{abstract}

\section{Introduction}

Reprogramming mature somatic cells into human induced pluripotent stem cells (hiPSCs) opened a new strategy in tissue engineering and regenerative medicine. The delivery of transcription factors (i.e., OCT4, SOX2, KLF4, and c-Myc) transits somatic cells into a state similar to embryonic stem cells. The pluripotency and unlimited proliferation makes hiPSCs an ideal cell source for the production of transplantable regenerative cell sources.

In the early years of hiPSC generation, skin fibroblasts were usually used for reprogramming. The first hiPSCs generated by Yamanaka himself was generated from human skin dermal fibroblasts (DF). DFs may be convenient to cultivate in vitro; however, it can be obtained only through an invasive punch biopsy procedure. Somatic cells that are easy to obtain from an individual were required as a substitute. Today, hiPSCs are generated from various cell sources such as blood cells, keratinocytes, urine cells, and more [1-3].

Previous reports suggest that the original primary cell source of hiPSCs can subsequently affect the in vitro differentiation ability. Some report that the differentiation ability is biasing the cells towards the tissue of origin [4-8]. Epigenetic memory such as DNA methylation is thought to be responsible for the different differentiation capacity $[9,10]$.

Through our previous studies, we successfully generated hiPSCs from peripheral blood mononuclear cells (PBMCs) and cord blood mononuclear cells (CBMCs) $[11,12]$. We also generated hiPSCs using fibroblast-like synoviocytes (FLSs) isolated from the synovium in the knee joint of osteoarthritis (OA) patients [13, 14]. CBMC-derived hiPSCs were generated with CBMCs with homozygous human leukocyte antigen (HLA) types for future use in regenerative 
medicine. Using the CBMC-derived hiPSCs, we confirmed the potential in chondrogenic differentiation by generating chondrogenic pellets that are about $1-2 \mathrm{~mm}$ in size $[15,16]$. After 21 days of differentiation using CBMC-hiPSCs in chondrogenic differentiation medium containing human BMP2 and TGF $\beta 3$, the chondrogenic pellets showed increased expression of chondrogenic markers (i.e., ACAN, COL2A1, COMP, and SOX9). Extracellular matrix (ECM) proteins were positively detected in the CBMC-hiPSC-derived chondrogenic pellets and the quality was comparable with that generated using actual mesenchymal stem cells (MSCs), which is a generally used cell source for in vitro chondrogenesis.

It is well known that the adult articular cartilage lacks natural healing ability [17-20]. Chondrogenesis using hiPSCs was attempted for several years with hiPSCs generated from various origin cells (i.e., adipose-derived stem cells, fibroblasts, and articular chondrocytes) [15, 21-25]. All cell sources successfully went through chondrogenesis. However, to our knowledge, the capacity of chondrogenic differentiation between hiPSCs generated from different origins was not confirmed. This study aims to compare the chondrogenic efficacy of hiPSCs generated from PBMCs, DFs, FLSs, and CBMCs.

\section{Materials and Methods}

2.1. Dermal Fibroblast and Fibroblast-Like Synoviocyte Isolation and Maintenance. Skin samples were extracted by skin punch biopsy procedures. Synovium samples were received by arthroscopic synovectomy or total knee replacement surgery. The delivered skin and synovium was chopped and homogenized. Chopped tissues were resuspended in Dulbecco's modified Eagle's medium (DMEM, Gibco, Carlsbad, CA, USA) containing with $0.01 \%$ collagenase. Tissues were digested with collagenase for 4 hours at $37 \%$ with vigorous shaking. Cells were washed and resuspended in DMEM supplemented with $20 \%$ fetal bovine serum (FBS, Gibco) and 1\% penicillin/streptomycin solution (Gibco). Cells were cultured until more than $80 \%$ confluence was achieved.

2.2. Peripheral Blood and Cord Blood Mononuclear Cell Isolation and Maintenance. Blood was delivered in heparin tubes. Fresh blood was diluted with phosphate buffered saline (PBS) and placed onto Ficoll-Paque reagent. The samples were centrifuged for 30 minutes at $850 \times \mathrm{g}$. Isolated PBMCs were transferred to a new tube and washed with PBS. Cell pellet was resuspended in StemSpan media (STEMCELL Technologies, Vancouver, Canada) containing CC110 cytokine (STEMCELL Technologies) for expansion. Cells were incubated for 5 days before reprogramming. Fresh media was added if needed.

2.3. hiPSC Generation Using Dermal Fibroblasts and Synoviocytes. Cells were washed and treated with trypsin/ EDTA. Cells were counted and $3 \times 10^{5}$ cells per well was obtained. Cells were then resuspended in 20\% DMEM and seeded into a 6-well plate. Yamanaka factors were delivered by sendai virus (Invitrogen, Carlsbad, CA, USA). The next day, prealiquoted sendai virus was treated to the cells and incubated in $5 \% \mathrm{CO}_{2}, 37^{\circ} \mathrm{C}$ for 48 hours. After 48 hours, virus-containing media were removed and changed every other day for 6 days. On day 7, media were changed to hiPSC media. Cells were cultured until colonies appeared. Media were changed daily after they were transited to E8 media.

2.4. iPSC Induction Using Peripheral Mononuclear Blood Cells and Cord Blood Mononuclear Cells. Reprogramming and characterization was performed as previously described [12]. Cells transfected with sendai virus containing Yamanaka factors were maintained in a vitronectin-coated dish (Thermo Fisher Scientific, Waltham, MA, USA), and media were changed daily with fresh E8 medium (STEMCELL Technologies) before use. Cells were maintained in vitronectin-coated dishes. Media were changed daily.

2.5. Chondrogenic Differentiation Using Pellet Culture. The protocol was followed by the procedure shown in our previous studies $[15,16]$. A $1: 1$ mixture of TeSR-E8 and AggreWell media (STEMCELL Technologies) was added to $2 \times 10^{6}$ hiPS cells for EB generation. EBs were maintained in E8 media for 3 days and then in E7 media for additional 3 days. EBs were harvested and resuspended in outgrowth (OG) cell induction media consisted of DMEM, 20\% FBS, and $10 \%$ penicillin/streptomycin. EBs were counted and 50-70 EB per $\mathrm{cm}^{2}$ was seeded onto a gelatin-coated dish. OG cells were induced from the attached EBs for 3 days at $5 \% \mathrm{CO}_{2}, 37^{\circ} \mathrm{C}$. Next, cells were detached and the remaining EB clumps were removed using a $40 \mu \mathrm{m}$ cell strainer (BD Technologies, Franklin Lakes, NJ, USA). Single OG cells were harvested and plated onto a new gelatin-coated dish (1$5 \times 10^{4}$ cell per $\mathrm{cm}^{2}$ ). Cells were used up to passage 5 . OG cells were counted and $3 \times 10^{5}$ cells per pellet were prepared. Cells were harvested in a $15 \mathrm{~mL}$ conical tube and media were changed into chondrogenic differentiation media (CDM; DMEM supplemented with $20 \%$ knockout serum replacement, $1 x$ nonessential amino acids, $1 \mathrm{mM}$ L-glutamine, $1 \%$ sodium pyruvate, $1 \%$ ITS + Premix, $10^{-7} \mathrm{M}$ dexamethasone, $50 \mathrm{mM}$ ascorbic acid, and $40 \mu \mathrm{g} / \mathrm{mL}$ L-proline) supplemented with $10 \mathrm{ng} / \mathrm{mL}$ recombinant human bone morphogenetic protein 2 (BMP2), and transforming growth factor beta 3 (TGF $\beta 3$ ). Cells resuspended in CDM were centrifuged at $750 \times \mathrm{g}$ for 5 minutes. Generated pellets were maintained for 21 days and media were changed every 3 days.

2.6. Polymerase Chain Reaction. Chondrogenic pellets or cells were harvested and stored at $-80^{\circ} \mathrm{C}$. Samples were snap frozen with liquid nitrogen and ground using a pestle. Ground samples were incubated with TRIzol (Thermo Fisher Scientific), and mRNA was extracted according to the manufacturer's instructions. Using RevertAid ${ }^{\mathrm{TM}}$ First Strand cDNA Synthesis kit (Thermo Fisher Scientific), $2 \mu \mathrm{g}$ of the extracted total RNA was used to synthesize cDNAs. Real-time PCR reactions included $2 \mu \mathrm{L}$ of diluted cDNA ( $1: 10$ dilution). Real-time PCR was carried out using LightCycler ${ }^{\circledR} 480$ Instrument II (Roche, Basel, Switzerland). The geometric mean of GAPDH was used as an internal control to normalize the results. 
TABLE 1: Primers used for real-time PCR against pluripotent markers and chondrogenic markers.

\begin{tabular}{|c|c|c|c|c|c|}
\hline Description & Target name & REFSEQ_ID & Direction & Primer sequence & Size \\
\hline \multirow{8}{*}{ Pluripotency marker } & \multirow{2}{*}{ OCT4 } & \multirow{2}{*}{ NM_203289.5 } & Forward & GGGAAATGGGAGGGGTGCAAAAGAGG & \multirow{2}{*}{151} \\
\hline & & & Reverse & TTGCGTGAGTGTGGATGGGATTGGTG & \\
\hline & \multirow{2}{*}{ KLF4 } & \multirow{2}{*}{ NM_004235.4 } & Forward & TTCCCATCTCAAGGCACAC & \multirow{2}{*}{158} \\
\hline & & & Reverse & GGTCGCATTTTTGGCACT & \\
\hline & \multirow{2}{*}{ SOX2 } & \multirow{2}{*}{ NM_003106.3 } & Forward & GGGAAATGGGAGGGGTGCAAAAGAGG & \multirow{2}{*}{151} \\
\hline & & & Reverse & TTGCGTGAGTGTGGATGGGATTGGTG & \\
\hline & \multirow{2}{*}{ NANOG } & \multirow{2}{*}{ NM_024865.2 } & Forward & AAAGGCAAACAACCCACT & \multirow{2}{*}{270} \\
\hline & & & Reverse & GCTATTCTTCGGCCAGTT & \\
\hline \multirow{8}{*}{ Viral vector detection marker } & \multirow{2}{*}{$\mathrm{SeV}$} & & Forward & GGATCA CTA GGTGATATCGAGC & \multirow{2}{*}{181} \\
\hline & & & Reverse & ACCAGACAAGAGTTTAAGAGATATGTATC & \\
\hline & \multirow{2}{*}{ KOS } & & Forward & ATGCACCGCTACGACGTGAGCGC & \multirow{2}{*}{528} \\
\hline & & & Reverse & ACCTTGACAATCCTGATGTGG & \\
\hline & \multirow{2}{*}{ Klf4 } & & Forward & TTCCTGCATGCCAGAGGAGCCC & \multirow{2}{*}{410} \\
\hline & & & Reverse & AATGTATCGAAGGTGCTCAA & \\
\hline & \multirow{2}{*}{$\mathrm{c}-\mathrm{Myc}$} & & Forward & TAACTGACTAGCAGGCTTGTCG & \multirow{2}{*}{532} \\
\hline & & & Reverse & TCCACATACAGTCCTGGATGATGATG & \\
\hline \multirow{20}{*}{ Chondrogenic marker } & \multirow{2}{*}{ SOX9 } & \multirow{2}{*}{ NM_000346 } & Forward & GACTTCCGCGACGTGGAC & \multirow{2}{*}{99} \\
\hline & & & Reverse & GTTGGGCGGCAGGTACTG & \\
\hline & \multirow{2}{*}{ SOX5 } & & Forward & CAGCCAGAGTTAGCACAATAGG & \\
\hline & & NM_001261415.2 & Reverse & CTGTTGTTCCCGTCGGAGTT & 104 \\
\hline & & & Forward & GGATGCAATGACCCAGGATTT & \\
\hline & $50 \times 6$ & NM_033326.3 & Reverse & TGAATGGTACTGACAAGTGTTGG & 141 \\
\hline & & & Forward & TCGAGGACAGCGAGGCC & \\
\hline & ACAN & NM_001135.3 & Reverse & TCGAGGGTGTAGCGTGTAGAGA & 85 \\
\hline & & & Forward & GGCAATAGCAGGTTCACGTACA & \\
\hline & COL2A1 & NM_001844 & Reverse & CGATAACAGTCTTGCCCCACTTA & 79 \\
\hline & & & Forward & GATCCCCAAGGTGTCAGAGAAG & \\
\hline & CHAD & NM_001267 & Reverse & GCCAGCACCGGGAAGTT & 66 \\
\hline & & & Forward & AAAGTCAGCACATCTCCCAAG & \\
\hline & PRG4 & NM_005807.4 & Reverse & GTGTCTCTTTAGCGGAAGTAGTC & 199 \\
\hline & & & Forward & TCTGCGACAACGGCAAGGTG & \\
\hline & COL1A1 & NM_000088.3 & Reverse & GACGCCGGTGGTTTCTTGGT & 146 \\
\hline & & & Forward & CAGGCATAAAAGGCCCAC & \\
\hline & COL10A1 & NM_000493.3 & Reverse & GTGGACCAGGAGTACCTTGC & 108 \\
\hline & RIJU? & NM 001024630 & Forward & CCAGATGGGACTGTGGTTACTG & 65 \\
\hline & RUNX2 & NM_001024630 & Reverse & TTCCGGAGCTCAGCAGAATAA & 65 \\
\hline Housekeening & GAPDH & NM 0020465 & Forward & ACССАСТССТССАССТТTGA & 101 \\
\hline Housekeeping gene & GAPDH & NM_002046.5 & Reverse & CTGTTGCTGTAGCCAAATTCGT & 101 \\
\hline
\end{tabular}

The detection of viral vectors was followed by the manufacturer's instruction produced in the reprogramming sendai virus kit (Invitrogen). Reverse transcription reaction was performed with the synthesized cDNAs. Integration of viral vectors was confirmed using four primers; $\mathrm{SeV}, \mathrm{KOS}$, KLF4, and c-Myc. The primer sequences are provided in Table 1.

2.7. Histological Analysis of Pellets. Chondrogenic pellets were washed with PBS. Pellets were fixed in $4 \%$ paraformaldehyde for 2 hours at room temperature (RT). Dehydration was performed with increasing sequential ethanol solutions. Additional clearing was done with sequential ethanolxylene mixtures and pellets were infiltrated with paraffin overnight. Paraffin blocks were fixed and $7 \mu \mathrm{m}$ sections were obtained using a microtome. Before staining the sections, slides were placed in a $60^{\circ} \mathrm{C}$ oven for at least 10 minutes. Slides were immediately deparaffinized using xylene. Slides were rehydrated with decreasing sequential ethanol series and were rinsed with running tap water for 1 minute each. 
TABLE 2: Information of generated hiPSCs.

\begin{tabular}{lcccc}
\hline hiPSC type & No & Sex & Cell type & Description \\
\hline \multirow{2}{*}{ DF-hiPSC } & 1 & $\mathrm{~F}$ & & Donor number 1 \\
& 2 & $\mathrm{M}$ & Dermal fibroblast & Donor number 2 \\
& 3 & $\mathrm{M}$ & & Donor number 3 \\
\hline \multirow{3}{*}{ PBMC-hiPSC } & 1 & $\mathrm{~F}$ & & Donor number 1 \\
& 2 & $\mathrm{M}$ & Peripheral blood Mononuclear cell & Donor number 2 \\
& 3 & $\mathrm{~F}$ & & Donor number 4 \\
\hline \multirow{3}{*}{ FLS-hiPSC } & 1 & $\mathrm{~F}$ & & OA patient number 1 \\
& 2 & $\mathrm{~F}$ & & OA patient number 2 \\
& 3 & $\mathrm{~F}$ & & OA patient number 3 \\
\hline \multirow{3}{*}{ CBMC-hiPSC } & 1 & $\mathrm{~F}$ & Cord blood mononuclear cell & Donor number 5 \\
& 2 & $\mathrm{M}$ & & Donor number 6 \\
& 3 & $\mathrm{M}$ & & Donor number 7 \\
\hline
\end{tabular}

Toluidine blue staining was done by incubating the hydrated slides in $0.04 \%$ toluidine blue (Sigma Aldrich, St. Louis, Missouri, USA) solution for 10 minutes. Slides were washed in running tap water and dried for 10 minutes until complete dryness. After the staining process, slides were dehydrated with an increasing sequential ethanol series. Ethanol was cleared with 2 cycles of $100 \%$ xylene and slides were mounted with VectaMount ${ }^{\mathrm{TM}}$ Permanent Mounting Medium (Vector Laboratories, Burlingame, CA, USA).

2.8. Immunohistochemistry. Slides were placed in a $60^{\circ} \mathrm{C}$ oven for 10 minutes and deparaffinized with 2 cycles of xylene. Slides were rehydrated and were incubated in boiling citrate buffer (Sigma Aldrich) for antigen unmasking. After cooling the unmasked slides, endogenous peroxidase activity was blocked by treating the slides with $3 \%$ hydrogen peroxide (Sigma Aldrich). Slides were washed and blocked with trisbuffered saline (TBS) containing $1 \%$ bovine serum albumin (BSA). Primary antibodies were diluted in blocking solution (collagen type II, 1/100; collagen type I, 1/200; collagen type X, 1: 250, Abcam). Slides were incubated with diluted primary antibody at $37^{\circ} \mathrm{C}$ for 1 hour. Slides were washed with TBS containing $0.1 \%$ tween-20. Secondary antibodies (1/200; Vector Laboratories) diluted in blocking buffer were treated for 40 minutes at RT. After washing out the secondary antibody, slides were treated with $\mathrm{ABC}$ reagent drops (Vector Laboratories) for 30 minutes. DAB solution (Vector Laboratories) was followed and incubated for 5 minutes. Slides were washed and counterstained with Mayer's hematoxylin (Sigma Aldrich) for 1 minute. Slides were dehydrated and cleared. Slides were mounted and staining was confirmed under a bright-field microscope.

2.9. Statistical Analysis. Comparison of each of cell typederived chondrogenic pellets and hiPSCs was analyzed by Kruskal-Wallis one-way ANOVA followed by Dunn's multiple comparison test. Analysis was done using the software GraphPad Prism $5\left({ }^{*} P<0.05 ;{ }^{* *} P<0.01\right.$; and ${ }^{* * *} P<0.001$ for statistically significant differences).

\section{Results}

3.1. Generation of hiPSCs from a Different Origin. Human iPSCs were generated from DFs, PBMCs, FLSs and CBMCs. The information of each cell line is shown in Table 2. Somatic cells were obtained from three individual donors per cell type. Some of the PBMCs and DFs were obtained from the identical donor. FLSs were isolated from the joint of three osteoarthritis patient's synovium. Three hiPSC clones were generated from each hiPSC and three chondrogenic pellets were analyzed individually from each cell line.

Primary cells were isolated from skin tissue, blood, synovium, and cord blood (Figure 1(a)). Primary cells were transduced with sendai viral vectors containing Yamanaka reprogramming factors. After several passaging and purification, reprogrammed cells exhibited a colony with compact morphology (Figure 1(b)). Pluripotency of the generated hiPSCs was confirmed with several markers. Colonies of each cell line showed positive expression of OCT4 and TRA-1-60 (Figure 1(c)). The colonies were also positively stained against alkaline phosphatase (AP) (Figure 1(d)). By real-time PCR, the relative expression of OCT4, SOX2, NANOG, and KLF4 was assessed in hiPSCs generated from different somatic cells (Figures 1(e)-1(h)). Interestingly, the expression of pluripotent markers was higher in PBMC and CBMC primary cells. Also, KLF4 expression was comparably high in FLSs as well. All of the pluripotent markers were expressed highest in DF-hiPSCs. The tendency of each pluripotent marker showed an identical pattern of expression. The silencing of integration of reprogramming viral vectors was confirmed in the hiPS cell lines (Figure S1). We have confirmed that the generated hiPSCs were viral vector free. Taken all together, we successfully reprogrammed nonintegrated hiPSCs from DFs, PBMCs, FLSs, and CBMCs with confirmed pluripotency.

3.2. Chondrogenic Pellet Generation Using hiPSCs from a Different Origin. In our previous studies, we confirmed the chondrogenic differentiation ability of CBMC-hiPSCs $[15,16]$. The protocol we used is simply described in 

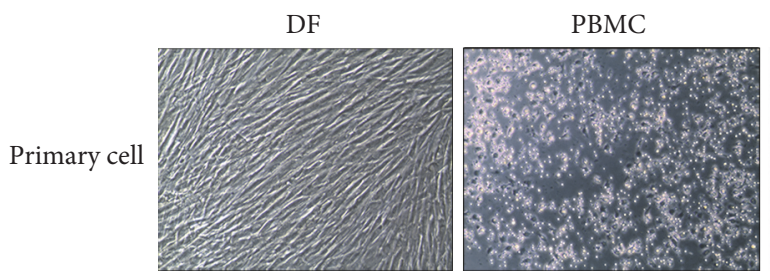

FLS
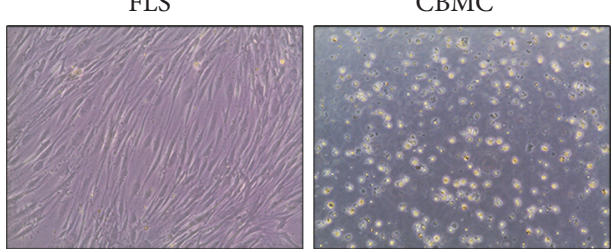

(a)
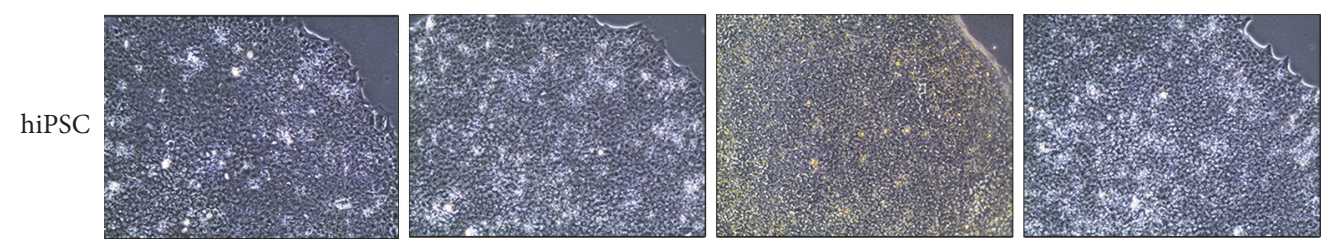

(b)
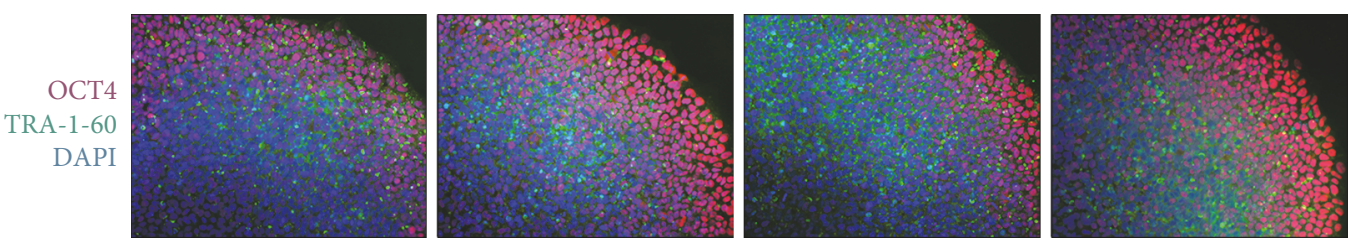

(c)
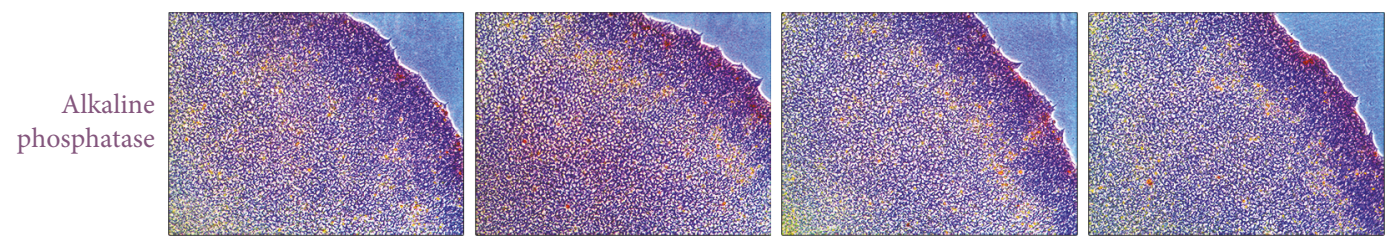

(d)

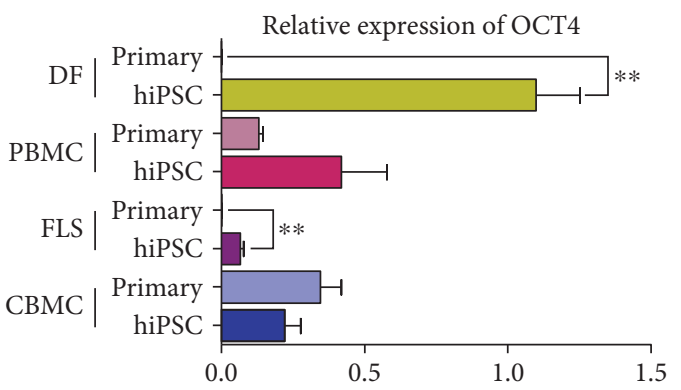

(e)

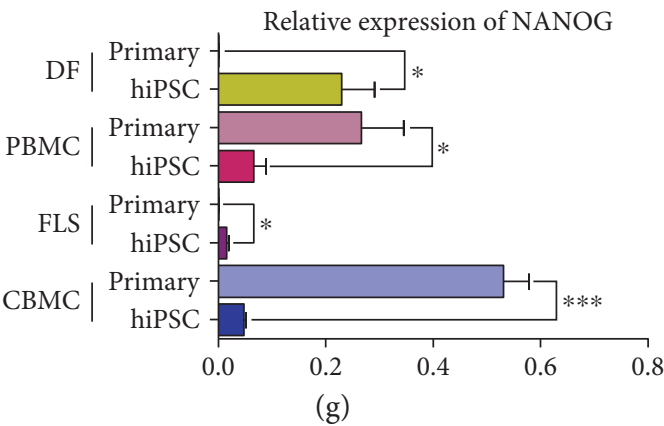

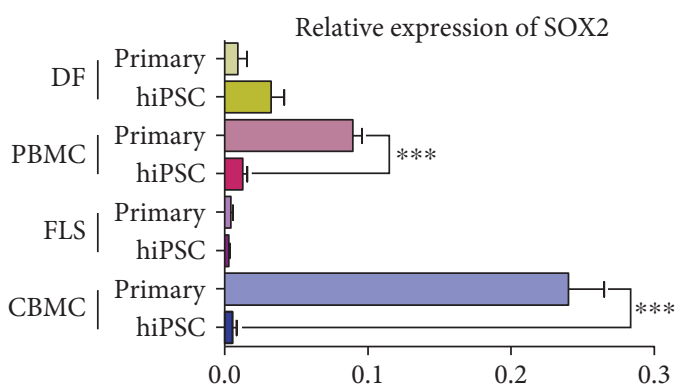

(f)

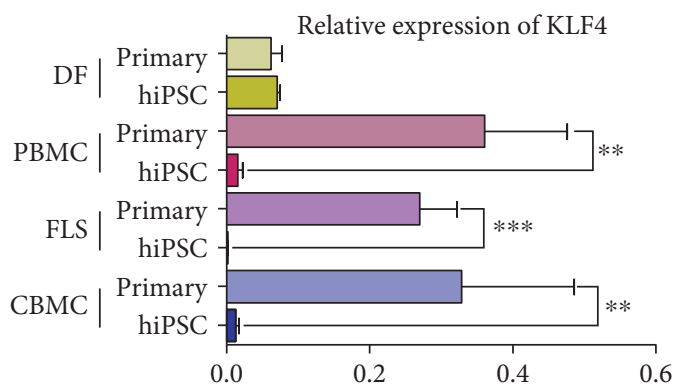

(h)

FIGURE 1: Characterization of DF-, PBMC-, FLS-, and CBMC-hiPSCs. (a) The morphology of each primary cell type: dermal fibroblast (DF), peripheral blood mononuclear cell (PBMC), fibroblast-like synoviocyte (FLS), and cord blood mononuclear cell (CBMC). (b) The morphology of the generated hiPSCs. (c) Immunohistology image of the hiPSC colonies stained against OCT4 and TRA-1-60. (d) Image of colonies stained against alkaline phosphatase (AS). (e) Relative expression of pluripotent markers in each cell line and primary cells $\left({ }^{*} P<0.05 ;{ }^{* *} P<0.01\right.$; and $\left.{ }^{* * *} P<0.001\right)$. 


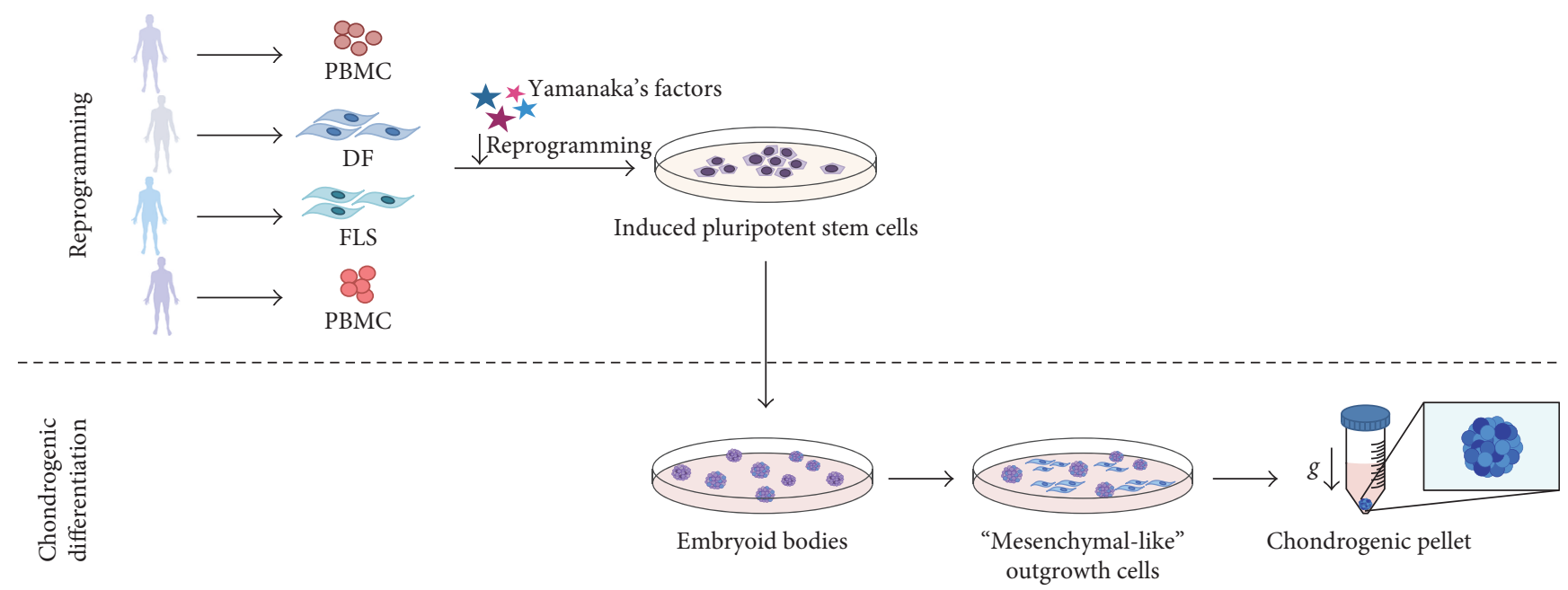

FIGURE 2: Scheme of experiment. Human iPSCs generated from different originated somatic cells were condensed into embryoid bodies (EB). EBs were attached to gelatin-coated dishes and outgrowth (OG) cells were induced. Using the OG cells, chondrogenic pellets were generated with centrifugal force and human growth factors.

Figure 2. Roughly, embryoid bodies (EBs) were generated using hiPSCs. EBs were cultivated for about 1 week and attached onto a gelatin-coated plate for outgrowth (OG) cell induction, which are cells that sprout out from the bottom of EBs. OG cells are known to have the similar characteristics of MSCs [26]. Koyama et al. referred them as "mesenchymal progenitor cells." Sprouted OG cells were then cultivated and used for chondrogenic differentiation. After 21 days of differentiation towards the chondrogenic lineage, pellets showed similar characteristics to that of cartilage (i.e., lacuna).

On day 3 of differentiation, OG cells derived from each hiPSC were condensed into a pellet (Figure 3(a)). OG cells derived from DF-hiPSCs and CBMC-hiPSCs condensed faster than the other two, and PBMC-hiPSCs took the longest time to form a pellet. After 21 days of differentiation, chondrogenic pellets were harvested (Figure 3(b)). CBMC- and DF-derived chondrogenic pellets had the largest size, indicating larger amounts of accumulated ECM proteins (Figure S2). On the other hand, FLS-derived pellets had the smallest size; however, there was no significant difference when the size were measured. ECM proteins were detected by toluidine blue staining (Figure 3(c)). Pellets generated from CBMC-hiPSCs showed high ECM accumulation through staining. PBMC- and DF-hiPSC-derived pellets also showed highly accumulated ECM proteins. However, FLS-hiPSC-derived pellets showed less accumulation. Cell death in the inner area was also detected, despite the small pellet size. The results of staining against collagen type II showed a similar tendency (Figure 3(d)). The staining in FLS-hiPSC-derived chondrogenic pellets showed hallow areas in the inner area. The other three cell types showed similar expression of collagen type II. The expression of collagen type I (Figure 3(e)) and type X (Figure 3(f)) was confirmed as well. The expression of both collagen types was highly detected in the inner area of the FLS-derived chondrogenic pellet. The other cell type-derived pellets had similar levels of collagen types I and X.
3.3. Chondrogenic Gene Expression in Chondrogenic Pellets Originating from Different Cell Source-Derived iPSCs. The gene expression of chondrogenic pellets generated from CBMC-derived hiPSCs $(n=27)$ was examined and compared with pellets derived from DF- $(n=27)$, PBMC- $(n=$ $27)$, and FLS-hiPSC- $(n=18)$ derived pellets. SOX9 is generally known as the transcriptional activator that is crucial for chondrogenesis. The expression of SOX9 was highest in CBMC-hiPSCs (Figure 4(a)). The expression was significantly higher than that of PBMC-hiPSCs or FLS-hiPSCs. SOX5 and SOX6 are closely related to the DNA-binding proteins that enhance the function of SOX9 [27]. The expression of SOX5 and 6 was also confirmed in the chondrogenic pellets. The expression of SOX5 in CBMC-hiPSC-derived pellets was significantly higher than DF- and FLS-hiPSCderived chondrogenic pellets (Figure 4(b)). PBMC-hiPSCderived pellets had similarly high expression of SOX5. This was also shown in SOX6 as well (Figure 4(c)). Aggrecan (ACAN) and collagen type II (COL2A1) are the major proteoglycan proteins in the articular cartilage that provides the hydrated gel structure. The expression of ACAN correlated to that of SOX9 (Figure 4(d)). ACAN expression was significantly higher in CBMC-hiPSC-derived chondrogenic pellets. Similar to SOX9, the expression pattern was not significant between DF-hiPSC-derived pellets and CBMChiPSC-derived pellets. Also, CBMC-hiPSC-derived pellets were the only type that showed significantly increased marker expression compared to its primary cell source. ACAN expression in PBMC- and FLS-hiPSC-derived pellets was relatively lower than the other two. In the case of COL2A1 expression, however, DF-hiPSC-derived pellets had the lowest expression (Figure 4(e)). Identical to ACAN expression, CBMC-hiPSC-derived pellets were the only type that showed significantly increased COL2A1 expression compared to its primary cell source. Lubricin (PRG4) is a proteoglycan that acts as lubricant in the joint. Interestingly, the expression of lubricin was significantly high in FLShiPSC-derived pellets (Figure 4(f)). On the other hand, 


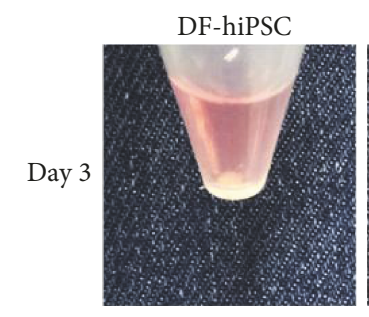

PBMC-hiPSC

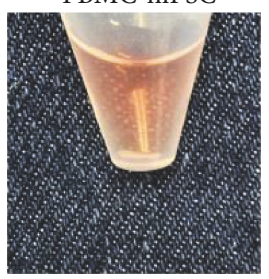

(a)
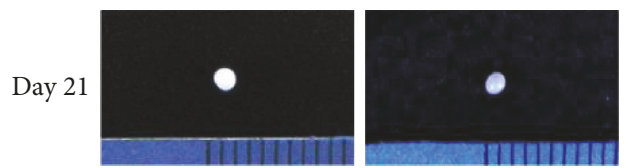

(b)
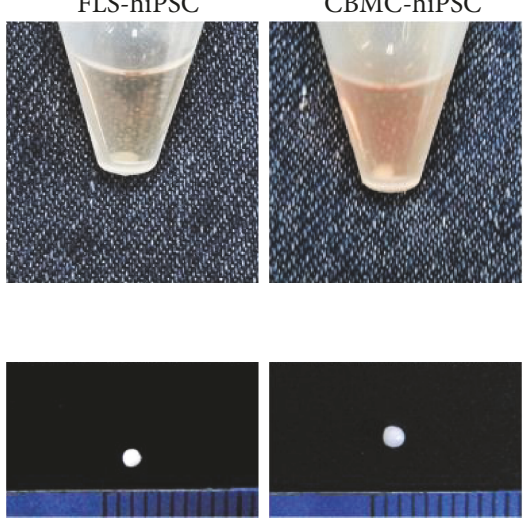

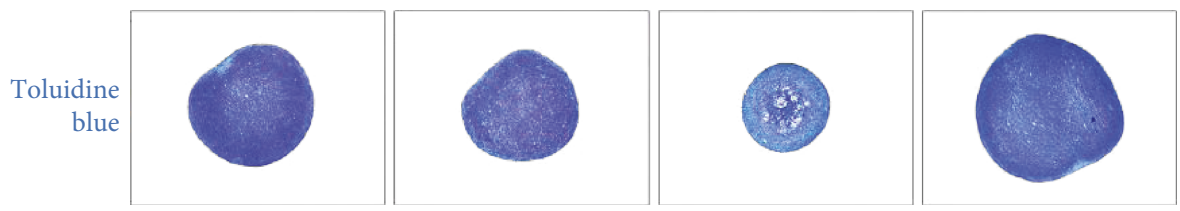

(c)

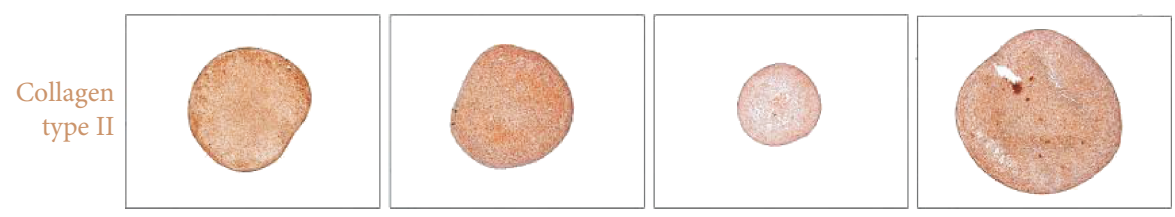

(d)

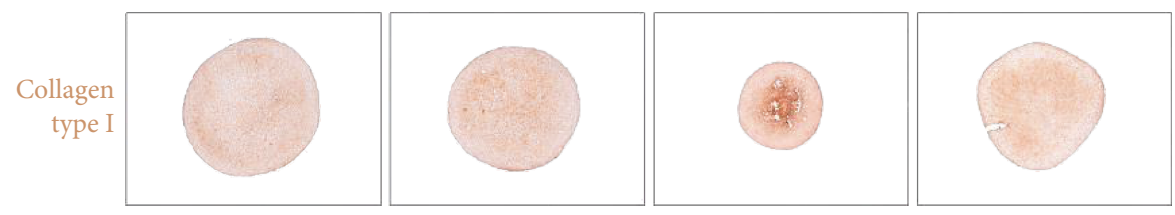

(e)

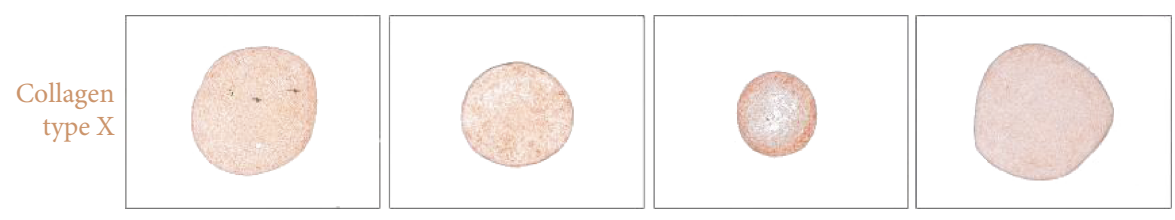

(f)

FIgURe 3: Chondrogenic pellets generated from hiPSC-derived from different originated somatic cells. (a) Morphology of outgrowth cells 3 days after differentiation. (b) Chondrogenic pellets after 21 days of chondrogenic differentiation. (c) Toluidine staining and (d) collagen type II immunohistology images of the chondrogenic pellets generated from each cell source-hiPSCs. (e) Collagen type I and (f) collagen type X staining images of chondrogenic pellets.

hiPSCs derived from PBMCs, FLSs, and CBMCs all had a relatively high expression of PRG4. Collagen type I (COL1A1) is a general marker for fibrocartilage, which is a less lubricated form of cartilage. As it is also a fibrotic marker, the expression was higher in FLS-hiPSC-derived pellets (Figure 4(g)). COL1A1 expression was high in both DF- and FLS-hiPSC-derived pellets. PBMC-hiPSC-derived pellets had the lowest expression of COL1A1 and FLShiPSC-derived pellets showed the highest expression. Yet, despite these differences, the results lacked significance.
Collagen type $\mathrm{X}$ (COL10A1) is a marker for hypertrophy. The expression of COL10A1 was mostly all lower than other markers (Figure 4(h)). Hypertrophy can lead to osteogenecity, and RUNX2 is another marker indicating early calcification or osteogenesis of cartilage. RUNX2 expression was the highest in DF-hiPSC-derived chondrogenic pellets (Figure 4(i)). The expression was significantly lower in PBMC-hiPSC-derived pellets compared to CBMC-hiPSCderived pellets. However, the expression of RUNX2 was relatively lower than all the other markers in every cell 
Early chondrogenic marker

SOX9

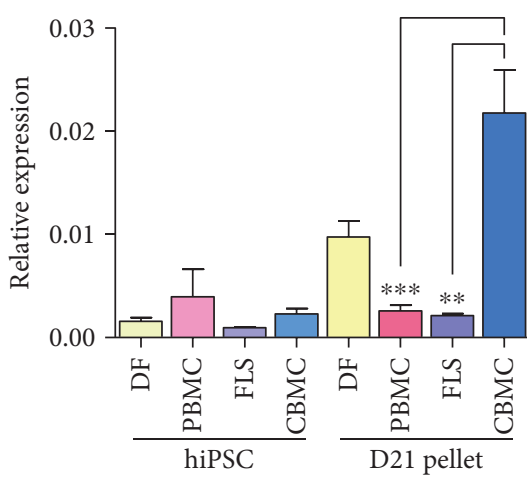

(a)

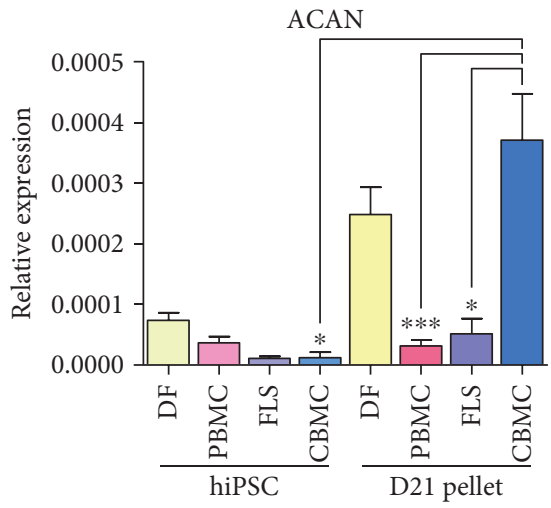

(d)

COL1A1

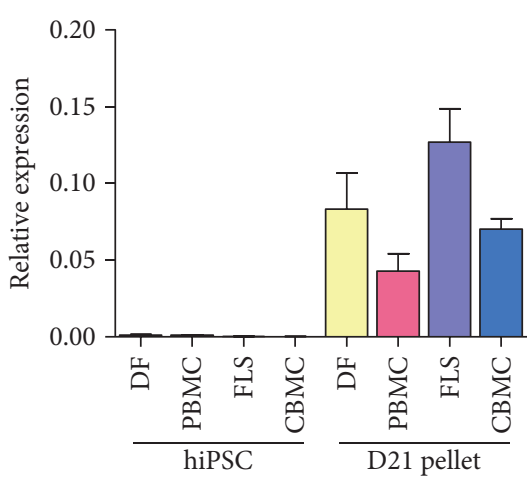

(g)

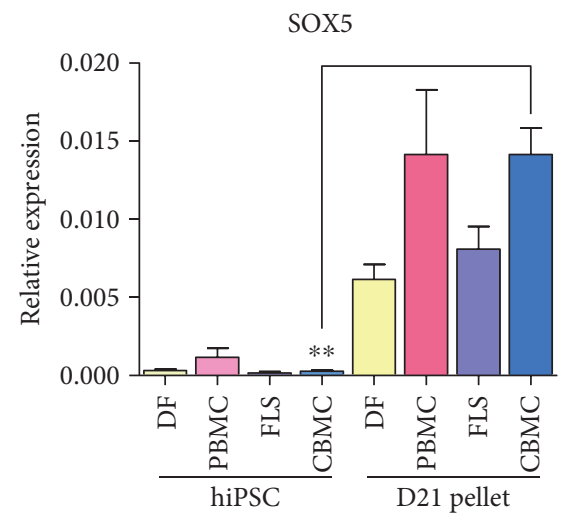

(b)

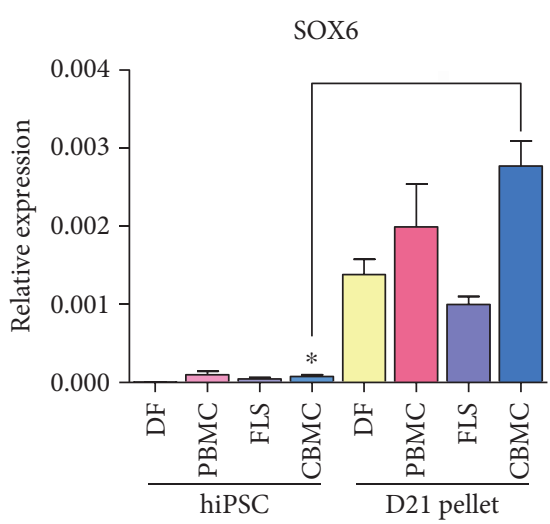

(c)

Cartilage matrix marker

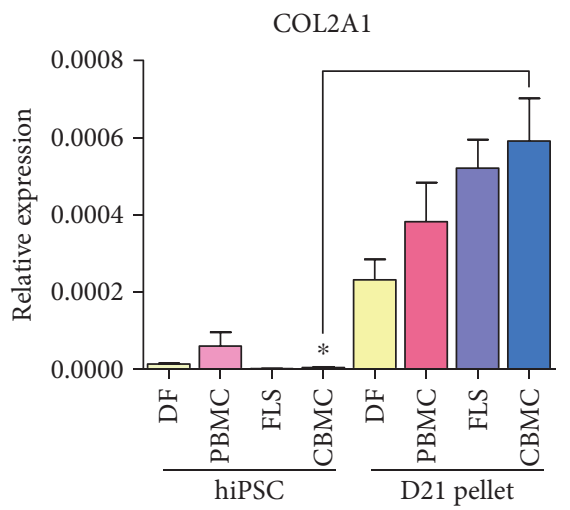

(e)

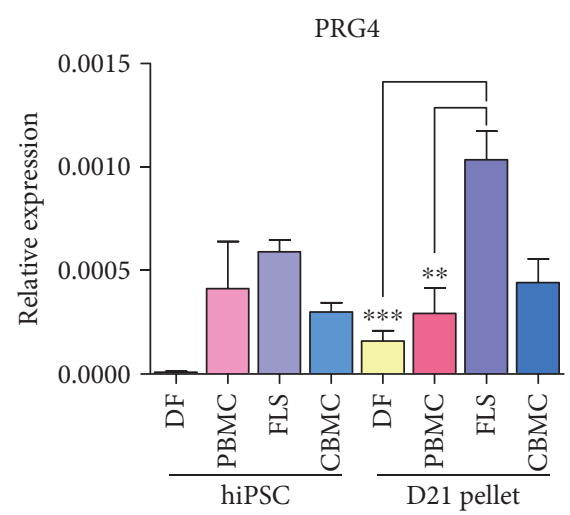

(f)

Hypertrophy and fibrotic marker

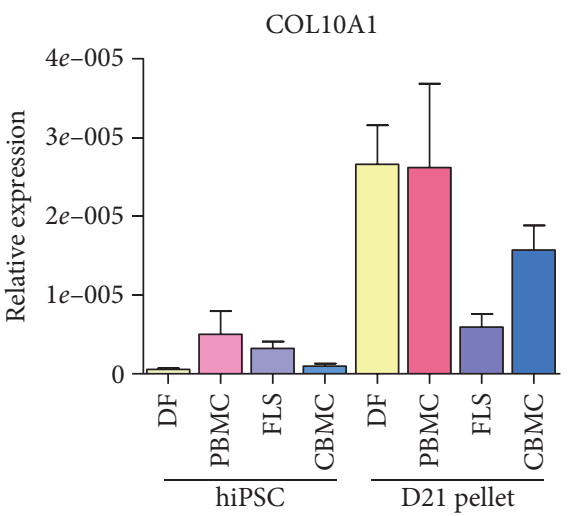

(h)

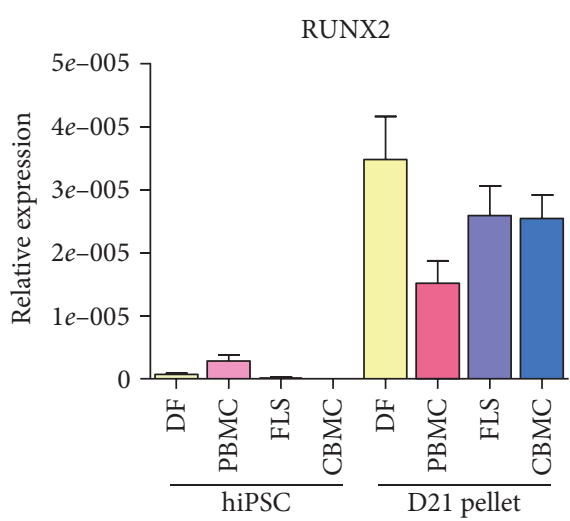

(i)

FIGURE 4: Gene expression in chondrogenic pellets. The expression of transcriptional activators or early chondrogenesis markers (a) SOX9, (b) SOX5, and (c) SOX6 was confirmed. The expression of matrix proteins, (d) aggrecan (ACAN), (e) collagen type II (COL2A1), and lubricin (PRG4) was confirmed. Also, hypertrophy or fibrotic markers such as (g) collagen type I (COL1A1), (h) collagen type X (COL10A1), and (i) RUNX2 was confirmed as well. To confirm the gene expression in each cell type, $n=9-18$ pellets were used in this experiment $\left({ }^{*} P<0.05 ;{ }^{* *} P<0.01\right.$; and $\left.{ }^{* * *} P<0.001\right)$.

type-derived chondrogenic pellets and it also lacks significance between all samples. The levels of pluripotent markers were measured in the generated chondrogenic pellets (Figure S3). The expression levels of OCT4, SOX2, and NANOG were all decreased in the generated pellets. The most important hiPSC marker, NANOG, was most significantly decreased in the CBMC-derived chondrogenic pellets. However, KLF4 expression was increased in all cases. Through these results, CBMC-hiPSC-derived pellets had a fairly superior quality than the other three cell sources. 
Yet, there was not much morphological difference between CBMC-, PBMC-, and DF-derived cell lines. The expression of pluripotent markers was also confirmed in chondrogenic pellets (Figure S3). The expression of hiPSC markers (i.e., OCT4, SOX2, and NANOG) was downregulated in differentiated chondrogenic pellets. However, the expression of KLF4 was increased in the chondrogenic pellets.

\subsection{Gene Expression in Pellets Generated from hiPSCs} Reprogrammed from Cells Sharing the Same Genetic Identity. Two of each of PBMC- and DF-hiPSCs were obtained from the same donor. To compare the chondrogenic potential from different cell sources (DFs and PBMCs) obtained from the same donor, we profiled the chondrogenic and other marker expression. Similar to the results of Figure 4(a), the expression of SOX9 was significantly higher in DF-hiPSC-derived pellets even in genetically identical conditions (Figure 5(a)). The expression of SOX5 turned out to be the opposite of the earlier data (Figure 4(b)); however, it was shown that the difference was not significant (Figure 5(b)). The expression of SOX6 was higher in PBMC-hiPSC-derived pellets as predicted; however, there was no significance as well (Figure 5(c)). The expression of ACAN was significantly higher in DF-hiPSC-derived chondrogenic pellets (Figure 5(d)). This was identical to the results shown in Figure 4(d). COL2A1 and PRG4 expression also showed a similar pattern as shown in Figure 4 (Figures 5(e) and 5(f)). The expression of COL1A1 was higher in DF-hiPSC-derived pellets (Figure 5(g)). COL10A1 expression was higher in PBMC-hiPSC-derived pellets (Figure 5(h)); however, the expression of RUNX2 was lower than DF-hiPSC-derived pellets. Taken all together, DF-hiPSC-derived pellets showed higher expression of the early transcription marker SOX9 and proteoglycan gene ACAN. Most of the markers had no significant difference in the chondrogenic pellets generated from PBMC- and DF-hiPSCs sharing the same genetic identity.

\section{Discussion}

In this present study, the chondrogenic differentiation potential of hiPSCs generated from different donor cell sources was investigated. Chondrogenic pellets were generated using several cell sources: CBMC, PBMC, DF, and FLSs. Between these samples, two of each of PBMCs and DFs were genetically identical. FLSs were obtained from an osteoarthritis patient's synovial tissue. The somatic cell source seemed to affect the chondrogenecity of hiPSCs. Our group previously showed the chondrogenic differentiation potential of CBMC-derived hiPSCs [15]. Compared to other cell sources, CBMC-hiPSC-derived pellets showed relatively higher expression of early and late (proteoglycan) markers of chondrogenesis. This data suggest that CBMC-hiPSCs can be used as a comparable cell source for in vitro chondrogenesis or cartilage regeneration.

In vitro chondrogenesis is an important issue, since the original tissue has limited capacity to regenerate or heal. Successful generation of hyaline cartilage in tissue engineering and in vitro cultivation systems can lead to tremendous therapeutic benefits in the related fields. Early in vitro chondrogenesis was done using MSCs or adipocyte-derived stem cells $[20,28]$. These two adult stem cells are also actually used in current clinics along with autologous chondrocytes for the regeneration of the damaged cartilage [29-31]. Despite their high chondrogenic potential, the low coverage of the cells was the limit of these cell sources. Also, long in vitro cultures to proliferate these cells have the possibility of hypertrophy and dedifferentiation of cells $[32,33]$. The low cellularity in elderly or patients may also impair the therapeutic effects of these cells [34]. Embryonic stem cells (ESCs) were mentioned for cartilage regeneration as an alternative; however, the ethical issues related to the embryo destructions are hard to overcome in several institutes. The emergence of hiPSCs was a breakthrough because of these reasons. The unlimited proliferation by self-renewal and high pluripotency was similar to that of ESCs, and the ethical issues were removed [35]. The spontaneous differentiation of hiPSCs also resulted in cartilage-like tissues, indicating the potential ability of hiPSCs in chondrogenesis.

The generation of hiPSCs can be theoretically done using any adult somatic cells. Various cell types (i.e., dermal fibroblast, blood cell, urine cell, and keratinocyte) were shown to reprogram successfully into hiPSCs [1, 3, 36-42]. Because of these reasons, hiPSCs are now widely used in the fields of disease modeling and drug screening that can replace the use of diseased animal models [43]. It is also the cutting edge material for tissue regeneration. However, the cell source used in reprogramming is now on the issue, since it might affect the outcome of reprogramming and regeneration. We generated hiPSCs from DFs, PBMCs, FLSs, and CBMCs. The generated hiPSCs had similar protein expression levels of pluripotent markers (i.e., OCT4 and TRA-1-60) (Figure 1), yet the genetic expression rather differed. The genetic expression levels of pluripotent markers such as OCT4, SOX2, NANOG, and KLF4 were highly expressed in DF-derived hiPSCs. PBMC-derived hiPSCs had the second highest expression and FLS-derived hiPSCs had the lowest. Interestingly, the expression of pluripotent markers was higher in the primary blood cells (PBMCs and CBMCs). This is thought to be caused by the effect of the maintenance media of suspension cells which can increase the hematopoietic stem cell population. Also, the expression of KLF4 in FLS-derived hiPSCs was highly expressed (Figure 1(h)). This fact was already confirmed in our previous report [13]. This finding is still being investigated in our other projects; however, a recent study reported that KLF4 is an inflammatory regulator in FLSs via IL-6 signaling [44].

While improved understandings and several protocols were developed in chondrogenic differentiation using hiPSCs, the selection of the donor somatic cell source used in reprogramming has become an important issue [25]. In this study, genetically identical PBMC- and DF-hiPSC were differentiated into chondrogenic pellets (Figure 5). Our results suggested that the two cell sources eventually had no significant difference in chondrogenic potential under the same genetic circumstances. The expression of SOX9 and ACAN correlated and were the only markers that shown 


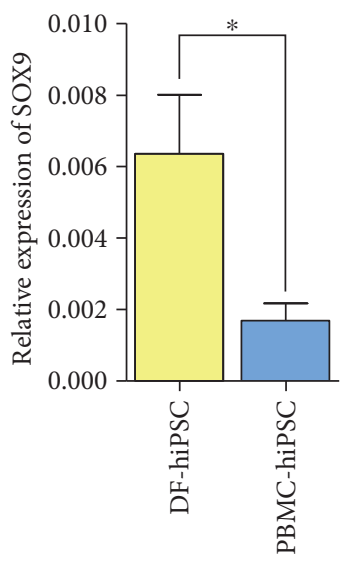

(a)

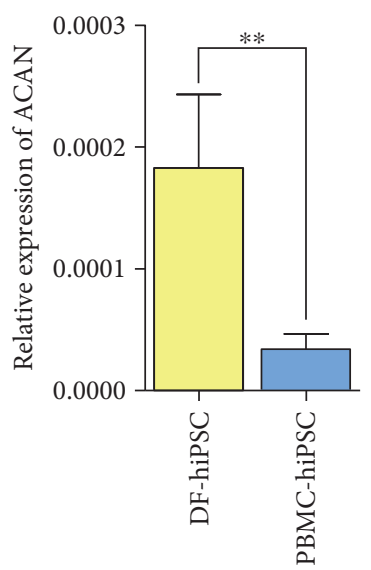

(d)

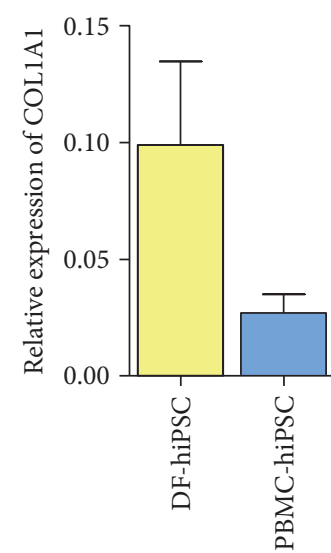

(g)

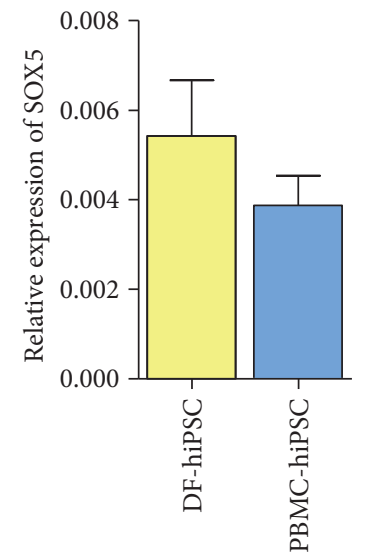

(b)

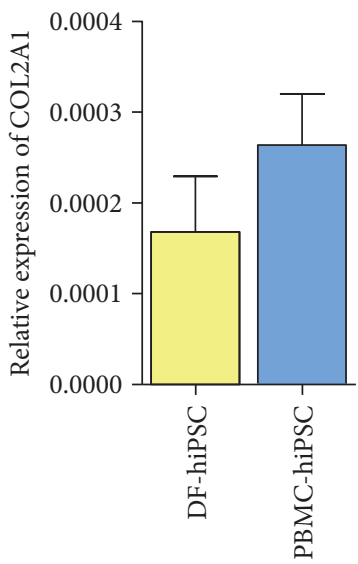

(e)

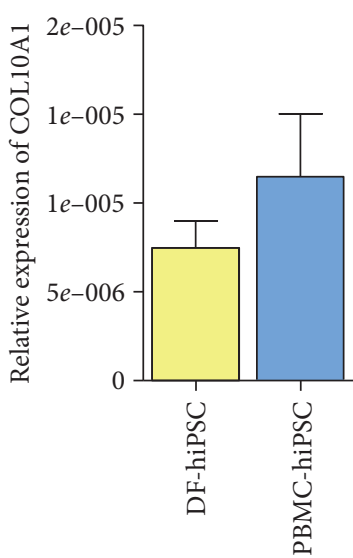

(h)

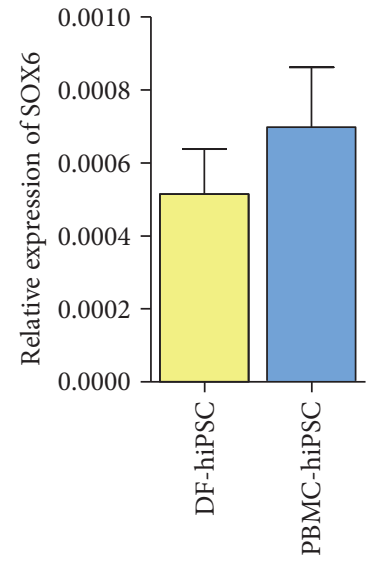

(c)

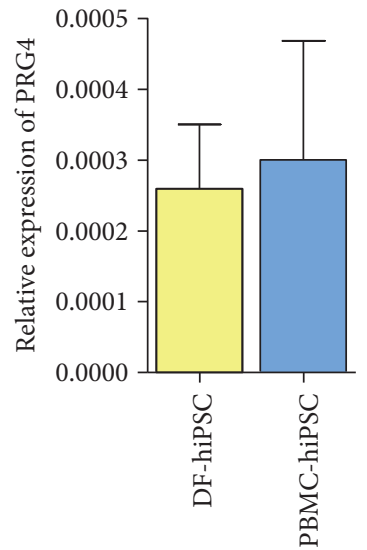

(f)

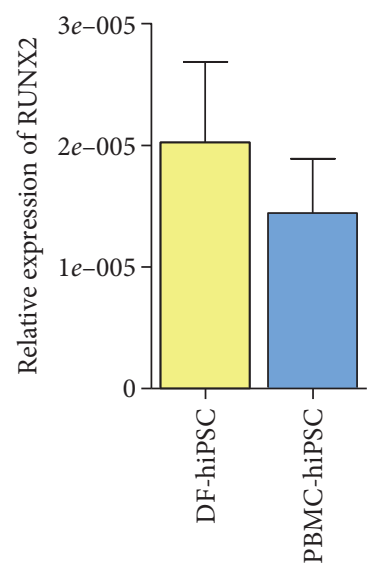

(i)

FIgURE 5: The expression of DF- and PBMC-hiPSC-derived chondrogenic pellets generated from the same donor. The expression of early transcription markers: (a) SOX9, (b) SOX5, and (c) SOX6. The expression of proteoglycan matrix proteins: (d) ACAN, (e) COL2A1, and (f) PRG4. The expression fibrotic and hypertrophic markers: (g) COL1A1, (h) COL10A1, and (i) RUNX2 $\left({ }^{*} P<0.05 ;{ }^{* *} P<0.01\right)$.

significant difference between the two differently originated hiPSCs. Both SOX9 and ACAN were significantly higher in DF-hiPSC-derived pellets. We concluded that some somatic cell sources might not have different outcomes in chondrogenesis. Yet, further examination and more cell lines that share the same genetic profiles to confirm chondrogenesis might be required before we assert these results.
The expression of pluripotent markers were examined in the chondrogenic pellets (Figure S3). The expression of OCT4, SOX2, and NANOG was decreased in chondrogenic pellets compared to hiPSCs. Interestingly, KLF4 expression was increased in all chondrogenic pellets generated from different cell types. This data can suggest that KLF4 might play an important role in chondrogenesis. The relation 
between KLF4 and chondrogenesis is not fully understood at this point; however, Outani and colleagues previously reported induction of chondrogenesis using KLF4 with other factors $[45,46]$. Chondrogenic cells were directly induced from mouse dermal fibroblasts by transducing only SOX9, c-Myc, and KLF4. This might explain the reason of the increased levels of KLF4 in cells undergoing chondrogenesis. Also, based on the fact that FLS-hiPSCs had high expression of KLF4 (Figure 1(h)), it might be a characteristic of the pathology of OA. Previous reports such as the one reported by Liu et al. suggest that KLF4 inhibit the expression of IL- $1 \beta$, which is the main cytokine that is thought to be closely related to OA. Further research on this subject may suggest another clue about the epigenetic memory of hiPSCs.

The inheritance of the initial epigenomes and characteristics of the primary cell source to the hiPSCs are mentioned as "epigenetic memory" [47]. This phenomenon makes hiPSC a reasonable source for disease modeling. It is thought that using patient-specific hiPSCs may reflect the pathological characteristics of the diseased somatic cell or individual. We also differentiated OA FLS-derived hiPSCs into chondrogenic pellets in this study. The pellets generated using FLS-derived hiPSCs showed low viability in the inner region, even though it had a relatively small size (Figure S2). The gene expression of OA FLS-hiPSC-derived chondrogenic pellets was also interesting. The expression of SOX9 was significantly lower in FLS-hiPSC-derived chondrogenic pellets (Figure 4(a)). The expression of the other two transcription factors, SOX5 and SOX6, was also low, compared to that of CBMC-hiPSC-derived chondrogenic pellets (Figures 4(b) and 4(c)). The expression of ACAN was also significantly lower than that DF- and CBMC-hiPSC-derived pellets. While the expression of COL2A1 and ACAN are usually known to have decreased expression in OA, however, COL2A1 expression was not that low than any other cell sources except CBMC-hiPSCs. Lubricin, or PRG4, is a protein in the cartilage ECM and synovial fluid that participates in the lubrication of the joint articular cartilage [48]. Interestingly, the expression of PRG4 was significantly higher than the other cell types. The expression of PRG4 was predicted to be decreased in OA; however, increased expression of PRG4 was also confirmed in OA patient human anterior cruciate ligament-MSCs [48]. Correlating with the previous reports, the high expression of PRG4 in OA might be an interesting subject to investigate. As other studies suggest that mechanical motions promote the expression of PRG4 in the articular cartilage, the high expression of PRG4 might be related to OA caused by external trauma or mechanical forces [49].

\section{Conclusions}

In conclusion, our CBMC-hiPSCs generated chondrogenic pellets with fair quality. However, no significant morphological difference was shown in normal hiPSC-derived pellets. With additional well-known benefits of the CBMCs (i.e., cell banking systems, stored HLA-typing information, and homozygous HLA types), the use of HLA-homozygous
CBMC-hiPSCs have several advantages for future use of hiPSCs in clinics and tissue transplantation [50]. Our study suggests CBMC-hiPSCs as an ideal source for future application in cartilage regeneration. Also, we suggest hiPSCs generated from different somatic cells with different genetic and epigenetic backgrounds as a tool to assess and understand chondrogenesis and the cartilage in vitro.

\section{Conflicts of Interest}

The authors declare that there is no conflict of interests.

\section{Acknowledgments}

This work was supported by a grant of the Korea Healthcare Technology R\&D Project, Ministry for Health, Welfare and Family Affairs, Republic of Korea (HI16C2177, HI16C2438, and HD16A1519).

\section{Supplementary Materials}

Supplementary 1. Figure S1: Confirmation of vector-free hiPSCs. The elimination of viral vectors was confirmed in generated hiPSCs. The expression was confirmed in clone \#1 of each hiPS cell line.

Supplementary 2. Figure S2: Size of chondrogenic pellets generated from hiPSCs. Chondrogenic pellets generated from CBMC-derived hiPSCs had the largest size.

Supplementary 3. Figure S3: The expression of pluripotent markers (OCT4, SOX2, NANOG, and KLF4) in day 21 chondrogenic pellets. The expression was compared to that of hiPSCs.

\section{References}

[1] T. Zhou, C. Benda, S. Duzinger et al., "Generation of induced pluripotent stem cells from urine," Journal of the American Society of Nephrology, vol. 22, no. 7, pp. 1221-1228, 2011.

[2] Y. H. Loh, S. Agarwal, I. H. Park et al., "Generation of induced pluripotent stem cells from human blood," Blood, vol. 113, no. 22, pp. 5476-5479, 2009.

[3] T. Aasen, A. Raya, M. J. Barrero et al., "Efficient and rapid generation of induced pluripotent stem cells from human keratinocytes," Nature Biotechnology, vol. 26, no. 11, pp. 1276-1284, 2008.

[4] J. Phetfong, A. Supokawej, M. Wattanapanitch, P. Kheolamai, Y. U-pratya, and S. Issaragrisil, "Cell type of origin influences iPSC generation and differentiation to cells of the hematoendothelial lineage," Cell and Tissue Research, vol. 365, no. 1, pp. 101-112, 2016.

[5] O. Bar-Nur, H. A. Russ, S. Efrat, and N. Benvenisty, "Epigenetic memory and preferential lineage-specific differentiation in induced pluripotent stem cells derived from human pancreatic islet beta cells," Cell Stem Cell, vol. 9, no. 1, pp. 17-23, 2011.

[6] K. Kim, A. Doi, B. Wen et al., "Epigenetic memory in induced pluripotent stem cells," Nature, vol. 467, no. 7313, pp. 285290, 2010.

[7] K. Kim, R. Zhao, A. Doi et al., "Donor cell type can influence the epigenome and differentiation potential of human induced 
pluripotent stem cells," Nature Biotechnology, vol. 29, no. 12, pp. 1117-1119, 2011.

[8] E. J. Kim, K. H. Kang, and J. H. Ju, “CRISPR-Cas9: a promising tool for gene editing on induced pluripotent stem cells," The Korean Journal of Internal Medicine, vol. 32, no. 1, pp. 42-61, 2017.

[9] Y. Ohi, H. Qin, C. Hong et al., "Incomplete DNA methylation underlies a transcriptional memory of somatic cells in human iPS cells," Nature Cell Biology, vol. 13, no. 5, pp. 541-549, 2011.

[10] M. Vitaloni, J. Pulecio, J. Bilic, B. Kuebler, L. Laricchia-Robbio, and J. C. Izpisua Belmonte, "MicroRNAs contribute to induced pluripotent stem cell somatic donor memory," Journal of Biological Chemistry, vol. 289, no. 4, pp. 20842098, 2014.

[11] Y. A. Rim, Y. Nam, and J. H. Ju, "Induced pluripotent stem cell generation from blood cells using Sendai virus and centrifugation," Journal of Visualized Experiments, no. 118, article e54650, 2016.

[12] Y. Kim, Y. A. Rim, H. Yi, N. Park, S. H. Park, and J. H. Ju, “The generation of human induced pluripotent stem cells from blood cells: an efficient protocol using serial plating of reprogrammed cells by centrifugation," Stem Cells International, vol. 2016, Article ID 1329459, 9 pages, 2016.

[13] J. Lee, Y. Kim, H. Yi et al., "Generation of disease-specific induced pluripotent stem cells from patients with rheumatoid arthritis and osteoarthritis," Arthritis Research \& Therapy, vol. 16, no. 1, article R41, 2014.

[14] Y. A. Rim, N. Park, Y. Nam, and J. H. Ju, "Generation of induced-pluripotent stem cells using fibroblast-like synoviocytes isolated from joints of rheumatoid arthritis patients," Journal of Visualized Experiments, no. 116, article e54072, 2016.

[15] Y. Nam, Y. A. Rim, S. M. Jung, and J. H. Ju, "Cord blood cell-derived iPSCs as a new candidate for chondrogenic differentiation and cartilage regeneration," Stem Cell Research \& Therapy, vol. 8, no. 1, p. 16, 2017.

[16] Y. Nam, Y. A. Rim, and J. H. Ju, "Chondrogenic pellet formation from cord blood-derived induced pluripotent stem cells," Journal of Visualized Experiments, no. 124, article e55988, 2017.

[17] J. A. Buckwalter, "Articular cartilage: injuries and potential for healing," Journal of Orthopaedic \& Sports Physical Therapy, vol. 28, no. 4, pp. 192-202, 1998.

[18] A. J. Sophia Fox, A. Bedi, and S. A. Rodeo, "The basic science of articular cartilage: structure, composition, and function," Sports Health: A Multidisciplinary Approach, vol. 1, no. 6, pp. 461-468, 2009.

[19] C. B. Carballo, Y. Nakagawa, I. Sekiya, and S. A. Rodeo, "Basic science of articular cartilage," Clinics in Sports Medicine, vol. 36, no. 3, pp. 413-425, 2017.

[20] Y. Jang, H. Jung, Y. Nam et al., "Centrifugal gravity-induced BMP4 induces chondrogenic differentiation of adiposederived stem cells via SOX9 upregulation," Stem Cell Research \& Therapy, vol. 7, no. 1, p. 184, 2016.

[21] J. Y. Ko, K. I. Kim, S. Park, and G. I. Im, "In vitro chondrogenesis and in vivo repair of osteochondral defect with human induced pluripotent stem cells," Biomaterials, vol. 35, no. 11, pp. 3571-3581, 2014.

[22] H. Nejadnik, S. Diecke, O. D. Lenkov et al., "Improved approach for chondrogenic differentiation of human induced pluripotent stem cells," Stem Cell Reviews and Reports, vol. 11, no. 2, pp. 242-253, 2015.

[23] N. Tsumaki, M. Okada, and A. Yamashita, "iPS cell technologies and cartilage regeneration,” Bone, vol. 70, pp. 48-54, 2015.

[24] A. Yamashita, M. Morioka, Y. Yahara et al., "Generation of scaffoldless hyaline cartilaginous tissue from human iPSCs," Stem Cell Reports, vol. 4, no. 3, pp. 404-418, 2015.

[25] R. M. Guzzo, V. Scanlon, A. Sanjay, R. H. Xu, and H. Drissi, "Establishment of human cell type-specific iPS cells with enhanced chondrogenic potential," Stem Cell Reviews and Reports, vol. 10, no. 6, pp. 820-829, 2014.

[26] N. Koyama, M. Miura, K. Nakao et al., "Human induced pluripotent stem cells differentiated into chondrogenic lineage via generation of mesenchymal progenitor cells," Stem Cells and Development, vol. 22, no. 1, pp. 102-113, 2013.

[27] C. F. Liu and V. Lefebvre, "The transcription factors SOX9 and SOX5/SOX6 cooperate genome-wide through superenhancers to drive chondrogenesis," Nucleic Acids Research, vol. 43, no. 17, pp. 8183-8203, 2015.

[28] L. Zhang, J. Hu, and K. A. Athanasiou, "The role of tissue engineering in articular cartilage repair and regeneration," Critical Reviews ${ }^{\mathrm{TM}}$ in Biomedical Engineering, vol. 37, no. 1-2, pp. 1-57, 2009.

[29] P. D. Gikas, L. Bayliss, G. Bentley, and T. W. R. Briggs, “An overview of autologous chondrocyte implantation," Journal of Bone and Joint Surgery - British Volume, vol. 91, no. 8, pp. 997-1006, 2009.

[30] M. Brittberg, A. Lindahl, A. Nilsson, C. Ohlsson, O. Isaksson, and L. Peterson, "Treatment of deep cartilage defects in the knee with autologous chondrocyte transplantation," The New England Journal of Medicine, vol. 331, no. 14, pp. 889895, 1994.

[31] L. Peterson, M. Brittberg, I. Kiviranta, E. L. Åkerlund, and A. Lindahl, "Autologous chondrocyte transplantation. Biomechanics and long-term durability," The American Journal of Sports Medicine, vol. 30, no. 1, pp. 2-12, 2002.

[32] B. L. Clair, A. R. Johnson, and T. Howard, "Cartilage repair: current and emerging options in treatment," Foot \& Ankle Specialist, vol. 2, no. 4, pp. 179-188, 2009.

[33] H. Chiang and C. C. Jiang, "Repair of articular cartilage defects: review and perspectives," Journal of the Formosan Medical Association, vol. 108, no. 2, pp. 87-101, 2009.

[34] K. Bobacz, L. Erlacher, J. Smolen, A. Soleiman, and W. B. Graninger, "Chondrocyte number and proteoglycan synthesis in the aging and osteoarthritic human articular cartilage," Annals of the Rheumatic Diseases, vol. 63, no. 12, pp. 1618$1622,2004$.

[35] K. Takahashi and S. Yamanaka, "Induction of pluripotent stem cells from mouse embryonic and adult fibroblast cultures by defined factors," Cell, vol. 126, no. 4, pp. 663-676, 2006.

[36] J. Wang, Q. Gu, J. Hao et al., "Generation of induced pluripotent stem cells with high efficiency from human umbilical cord blood mononuclear cells," Genomics, Proteomics \& Bioinformatics, vol. 11, no. 5, pp. 304-311, 2013.

[37] W. Wen, J.-P. Zhang, W. Chen et al., "Generation of integration-free induced pluripotent stem cells from human peripheral blood mononuclear cells using episomal vectors," Journal of Visualized Experiments, no. 119, article e55091, 2017.

[38] N. Nishishita, C. Takenaka, N. Fusaki, and S. Kawamata, "Generation of human induced pluripotent stem cells from 
cord blood cells," Journal of Stem Cells, vol. 6, no. 3, pp. 101108, 2011.

[39] X. Yulin, L. Lizhen, Z. Lifei et al., "Efficient generation of induced pluripotent stem cells from human bone marrow mesenchymal stem cells," Folia Biologica, vol. 58, no. 6, pp. 221-230, 2012.

[40] S. N. Dowey, X. Huang, B. K. Chou, Z. Ye, and L. Cheng, "Generation of integration-free human induced pluripotent stem cells from postnatal blood mononuclear cells by plasmid vector expression," Nature Protocols, vol. 7, no. 11, pp. 2013-2021, 2012.

[41] Y. D. Sohn, J. W. Han, and Y. S. Yoon, "Generation of induced pluripotent stem cells from somatic cells," Progress in Molecular Biology and Translational Science, vol. 111, pp. 1-26, 2012.

[42] D. S. Manoli, D. Subramanyam, C. Carey et al., "Generation of induced pluripotent stem cells from the prairie vole," PLoS One, vol. 7, no. 5, article e38119, 2012.

[43] S. Diecke, S. M. Jung, J. Lee, and J. H. Ju, "Recent technological updates and clinical applications of induced pluripotent stem cells," The Korean Journal of Internal Medicine, vol. 29, no. 5, pp. 547-557, 2014.

[44] X. Luo, J. Chen, J. Ruan et al., "Krüppel-like factor 4 is a regulator of proinflammatory signaling in fibroblast-like synoviocytes through increased IL-6 expression," Mediators of Inflammation, vol. 2016, Article ID 1062586, 13 pages, 2016.

[45] H. Outani, M. Okada, K. Hiramatsu, H. Yoshikawa, and N. Tsumaki, "Induction of chondrogenic cells from dermal fibroblast culture by defined factors does not involve a pluripotent state," Biochemical and Biophysical Research Communications, vol. 411, no. 3, pp. 607-612, 2011.

[46] H. Outani, M. Okada, A. Yamashita, K. Nakagawa, H. Yoshikawa, and N. Tsumaki, "Direct induction of chondrogenic cells from human dermal fibroblast culture by defined factors," PLoS One, vol. 8, no. 10, article e77365, 2013.

[47] S. Raab, M. Klingenstein, S. Liebau, and L. Linta, “A comparative view on human somatic cell sources for iPSC generation," Stem Cells International, vol. 2014, Article ID 768391, 12 pages, 2014.

[48] D. H. Lee, J. Ng, S. B. Kim, C. H. Sonn, K. M. Lee, and S. B. Han, "Effect of donor age on the proportion of mesenchymal stem cells derived from anterior cruciate ligaments," PLoS One, vol. 10, no. 3, article e0117224, 2015.

[49] H. Ogawa, E. Kozhemyakina, H. H. Hung, A. J. Grodzinsky, and A. B. Lassar, "Mechanical motion promotes expression of Prg4 in articular cartilage via multiple CREB-dependent, fluid flow shear stress-induced signaling pathways," Genes \& Development, vol. 28, no. 2, pp. 127-139, 2014.

[50] Y. A. Rim, N. Park, Y. Nam et al., "Recent progress of national banking project on homozygous HLA-typed induced pluripotent stem cells in South Korea," Journal of Tissue Engineering and Regenerative Medicine, pp. 1-6, 2017. 


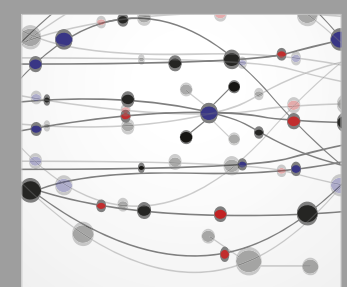

The Scientific World Journal
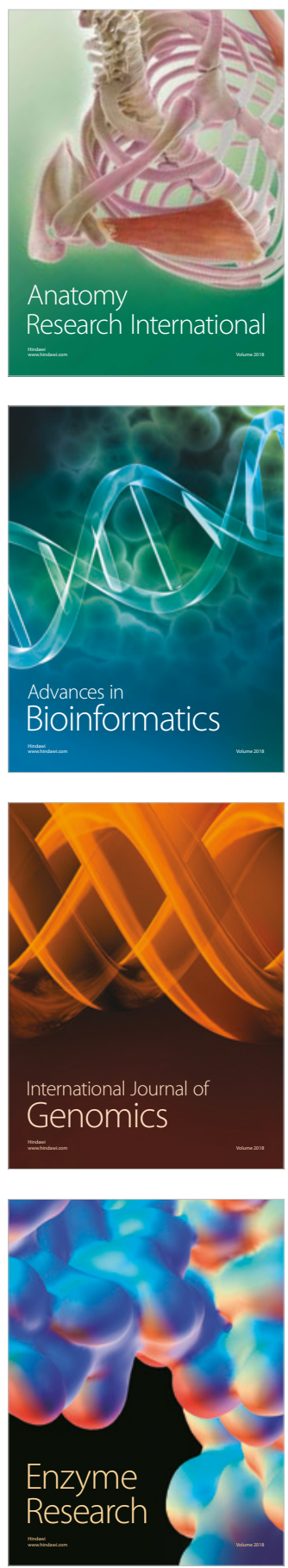
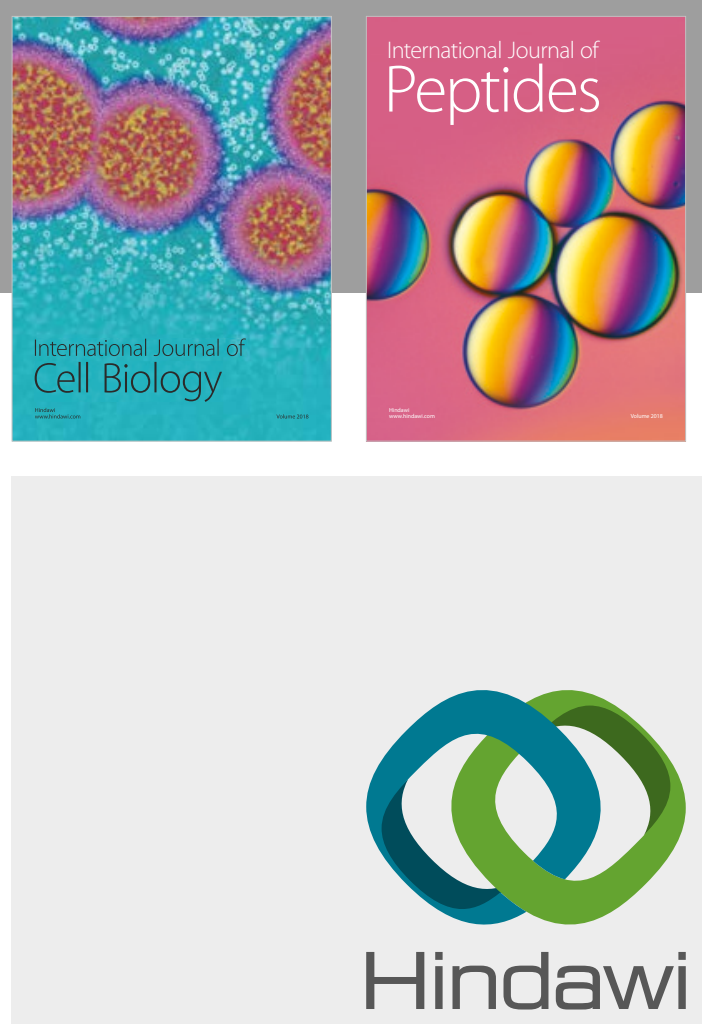

Submit your manuscripts at

www.hindawi.com
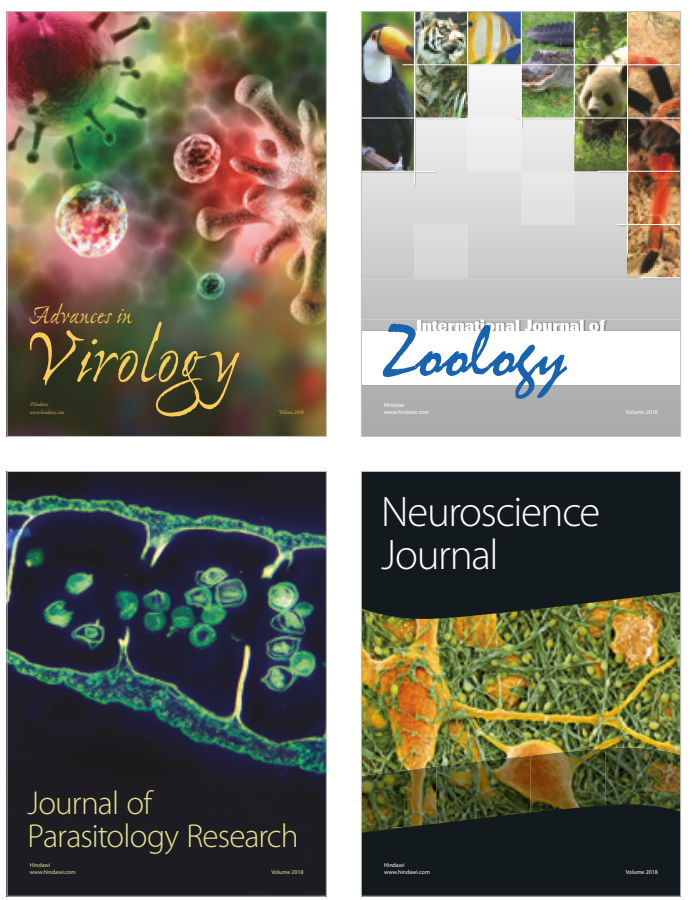
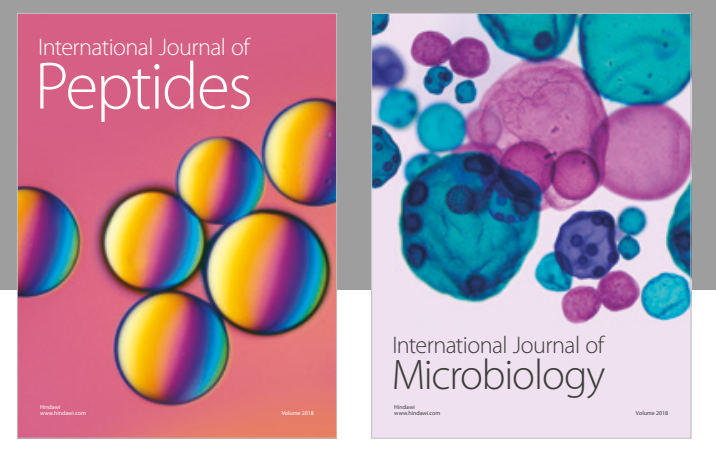

nternational Journal of Microbiology
Journal of
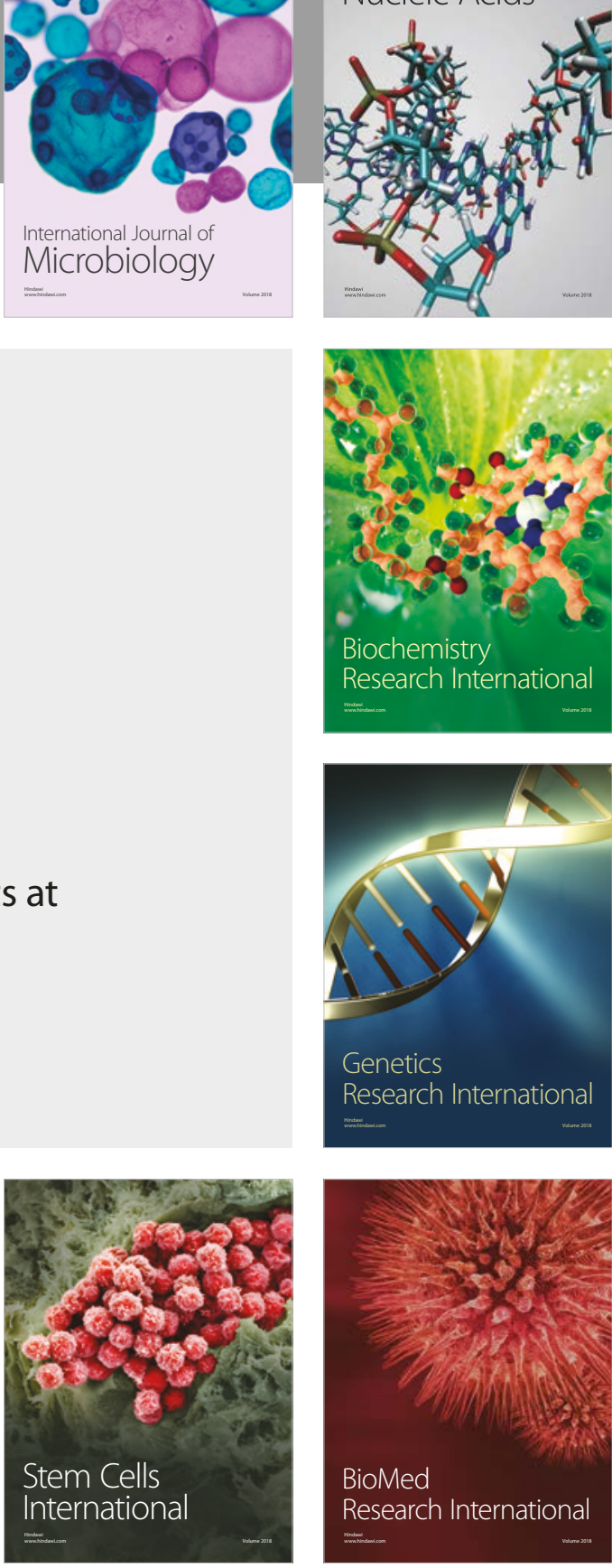
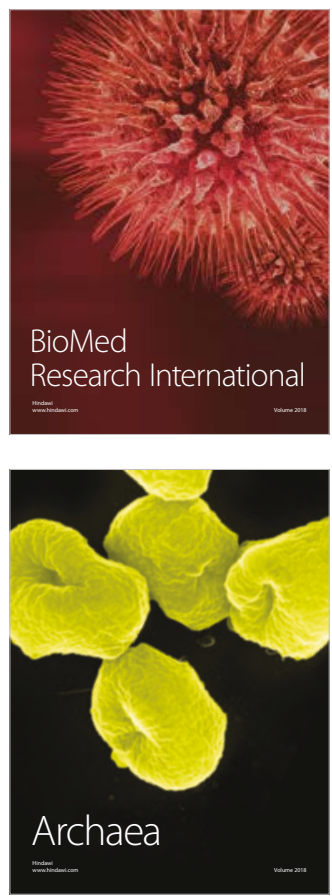